\title{
Current Research in Melanoma and Aggressive Nonmelanoma Skin Cancer
}

\author{
Reason Wilken, MD ${ }^{1}$ Maressa Criscito, MD ${ }^{1} \quad$ Anna C. Pavlick ${ }^{2}$ Mary L. Stevenson, MD ${ }^{1}$ \\ John A. Carucci, MD, PhD ${ }^{1}$ \\ ${ }^{1}$ Department of Dermatology, New York University Langone Health, \\ New York, New York \\ 2 Perlmutter Cancer Center, New York University Langone Health, \\ New York, New York \\ Address for correspondence Reason Wilken, MD, Department of \\ Dermatology, New York University Langone Health, 240 East 38th \\ Street, 11th Floor, New York, NY 10016-4576 \\ (e-mail: reason.wilken@nyulangone.org; reason.wilken@gmail.com).
}

Facial Plast Surg 2020;36:200-210.

\begin{abstract}
Keywords

- melanoma

- squamous cell carcinoma

- basal cell carcinoma

- immunotherapy

- PD-1

There have been several significant advances in cancer treatment in the last decade that are applicable to the treatment of melanoma and advanced nonmelanoma skin cancers. Among these are the development of immune checkpoint inhibitors targeting the programmed death protein-1 (PD-1)/programmed death legand-1 (PDL-1) axis, as well as targeted inhibitors of the BRAF/MEK signaling cascade in melanoma, and the hedgehog signaling pathway in basal cell carcinoma (BCC). These immune-based and targeted therapies have dramatically changed the treatment options for locally advanced and metastatic melanoma, Merkel's cell carcinoma, cutaneous squamous cell carcinoma ( $\mathrm{CSCC}$ ), and BCC. In this article, we will briefly review the currently approved targeted and immunotherapy-based treatments for locally advanced and metastatic melanoma, Merkel's cell carcinoma, and CSCC and discuss various combinations of approved therapies, as well as emerging therapeutic candidates that are currently in clinical trials, including novel checkpoint inhibitors in development, intratumoral oncolytic agents (viral and nonviral), and various immune-based therapies such as toll-like receptor (TLR) agonists, adoptive T-cell therapy, T-cell costimulation, and innate immune cell therapy. For advanced BCC, we will discuss trials investigating the currently approved smoothened (SMO) inhibitors for neoadjuvant use, emerging SMO inhibitors in development, topical SMO inhibitors, alternative targets in the hedgehog signaling pathway, and the use of anti-PD-1 agents for advanced BCC both alone and in combination with SMO inhibitors.
\end{abstract}

The development of immunotherapy (namely, checkpoint inhibitors targeting the programmed death protein-1 [PD-1]/programmed death legand-1 [PDL-1] pathway) and targeted molecular therapies in the last decade has dramatically changed the landscape for cancer therapeutics. Within cutaneous oncology, the advent of immune checkpoint inhibitors has revolutionized the treatment paradigms for locally advanced or metastatic melanoma, Merkel's cell carcinoma (MCC), and cutaneous squamous cell carcinoma (cSCC). In addition, insights into the molecular pathways of carcinogenesis have paved the way for development of

Issue Theme Management of Melanoma and Advanced Non-Melanoma Skin Cancers of the Face; Guest Editor: Jeffrey S. Moyer, MD multiple nonimmunologic targeted therapies including inhibitors of BRAF and MEK for melanomas harboring mutations in BRAF V600E/V600K and NRAS and inhibitors of the hedgehog signaling pathway that is constitutively activated in both familial and sporadic forms of basal cell carcinoma (BCC). In this article, we will briefly review the currently approved targeted and immunotherapy-based treatments for locally advanced and metastatic melanoma, MCC, CSCC, and BCC and discuss various combinations of approved therapies, as well as novel therapeutic candidates that are currently in clinical trials.
Copyright $\odot 2020$ by Thieme Medical Publishers, Inc., 333 Seventh Avenue, New York, NY 10001, USA. Tel: +1(212) 760-0888.
DOI https://doi.org/ 10.1055/s-0040-1709118. ISSN 0736-6825. 


\section{Melanoma}

\section{Currently Approved BRAF and MEK Inhibitors}

Vemurafenib is a small molecule inhibitor of the BRAF serine/threonine protein kinase that is found to be mutated in 40 to $60 \%$ of cutaneous melanomas (BRAF V600E/V600K) and was approved in 2011 for unresectable and metastatic melanoma harboring BRAF driver mutations. ${ }^{1}$ Dabrafenib is another BRAF inhibitor approved shortly after in 2013 for the treatment of late-stage BRAF-mutant melanoma. ${ }^{2}$ Development of resistance and rapid progression of disease has been observed with BRAF monotherapy due to compensatory MEK1/MEK 2 upregulation, leading to the approval of concurrent MEK inhibitors such as trametinib, cobimetinib, and bimimetanib for use in combination with BRAF inhibitors. ${ }^{2-4}$ Currently approved combinations include dabrafenib/trametenib, vemurafenib/cometinib, and encorafenib/binimetanib. ${ }^{5}$

\section{Currently Approved Immune Checkpoint Inhibitors}

Ipilimumab is a monoclonal antibody directed against the cytotoxic T-lymphocyte associated protein-4 (CTLA-4) T-cell receptor (TCR), and was approved in 2015 for adjuvant therapy of stage-III melanoma. ${ }^{6}$ CTLA- 4 is constitutively expressed on regulatory T-cells, as well as conventional T-cells after activation, and via interaction with B71/2 on antigen presenting cells leads to a downregulation of the immune response allowing tumor proliferation. ${ }^{7}$ The phase-III EORTC (European Organisation for Research and Treatment of Cancer) trial of ipilimumab $10 \mathrm{mg} / \mathrm{kg}$ compared with placebo leading to its approval showed significant improvement in recurrence-free survival (RFS) of $40.8 \%$ in the ipilumimab group versus $30.3 \%$ for placebo at the most recent 5-year follow-up; however, there was a high incidence (41.6\%) of immune-related grade- 3 and -4 events. $^{8}$ More recent trials have evaluated a lower-dose regimen of $3 \mathrm{mg} / \mathrm{kg}$, showing a decreased rate of grade 3 or higher toxicity (36.4 vs. 57\%) and no difference in RFS at 3-year follow up. ${ }^{9}$ However, with the introduction of immune checkpoint inhibitors targeting the PD-1 axis associated with improved efficacy and lower toxicity, adjuvant ipilimumab has fallen out of favor. ${ }^{5}$

PD-1 (also known as CD279) is a transmembrane protein receptor predominantly expressed on memory $\mathrm{T}$ cells, as well as B cells, monocytes, natural killer (NK) and dendritic cells that has an important role in regulating the immune response and maintaining self-tolerance through apoptosis of antigenspecific T-cells and upregulation of regulatory T-cells when activated by binding to its ligands PDL-1 (B7-H1) and PDL-2 (B7-DC). ${ }^{10} \mathrm{PDL}-2$ is primarily expressed on antigen-presenting cells (APCs), dendritic cells, and macrophages. ${ }^{10}$ PDL- 1 is expressed on multiple cell types including antigen-presenting cells, dendritic cells, B cells, monocytes, epithelial cells, as well as multiple types of cancer cells, including melanoma, lung, renal, and ovarian carcinomas, thus contributing to the ability of tumor cells in these immunologically active cancers to evade the immune system via activation of the PD-1/PDL-1 axis. ${ }^{11}$

Pembrolizumab and nivolumab are monoclonal antibodies against PD-1, and were approved in 2014 for unresectable or metastatic melanoma. ${ }^{12}$ Nivolumab was also approved in combination with ipilimumab for unresectable BRAF-wildtype melanoma in 2015, and approval of anti-PD-1 agents as for adjuvant treatment of stage-III melanoma following complete resection was granted for nivolumab in 2017 and pembrolizumab in 2019.

There are currently three commercially available PDL-1 inhibitors on the market, all of which are currently in clinical trials for melanoma (as detailed in below sections): atezolizumab, durvalumab, and avelumab. ${ }^{12}$ Atezolizumab was approved in 2016 for advanced urothelial carcinoma, and in 2019 for both advanced non-small-cell lung cancer (NSCLC), as well as advanced triple-negative breast carcinoma expressing PDL-1 (as demonstrated by the PD-L1 IHC22C3 pharmDx assay) in combination with paclitaxel. Durvalumab was approved in 2017 for advanced and metastatic urothelial carcinoma, and avelumab was approved in 2017 for metastatic MCC.

\section{Clinical Trials of Anti-PDL-1 Agents as Monotherapy in Melanoma}

Avelumab is currently in phase I (JAVELIN solid tumor trial, NCT01772004) for unresectable stage-IIIC/IV cutaneous and ocular melanoma that had progressed after at least one prior therapy for metastatic disease, and phase $1 \mathrm{~B}$ results were reported in early $2019 .^{13}$ The overall response rate was $21.6 \%$ (7.8\% of patients with complete response and $13.8 \%$ with a partial response). The mean progression-free survival (PFS) was 3.1 months, and mean overall survival (OS) time was 17.2 months. No grade- 4 treatment-related adverse events (TRAEs) or deaths occurred, and the rate of grade-3 TRAEs was $7.8 \%{ }^{13}$

Atezolizumab is slated to begin a phase-I trial (not yet recruiting) as a neoadjuvant agent prior to surgery for resectable stage IB-IIC melanoma meeting criteria for a sentinel lymph node biopsy (SLNB; NCT04020809).

\section{Combination and Sequential Trials with Immunotherapy and Targeted Therapy}

For patients with unresectable or metastatic BRAF-positive melanoma, the current therapeutic options include targeted therapies (BRAF/MEK inhibitor combinations), as well as antiPD-1 immunotherapies. While the addition of MEK inhibitors improved the durability of clinical responses to BRAF inhibition, development of resistance remains an issue and the response durations achieved with targeted therapy combinations are shorter than those achieved with systemic immunotherapeutic agents. ${ }^{14}$ The potential utility of combination trials is being investigated, as targeted agents induce higher initial response rates but lack durability, while the clinical response to PD-1 immunotherapy is often slower at onset but more durable. ${ }^{15}$ In addition, preclinical studies have demonstrated increased CD8 + T lymphocyte infiltration in the tumor microenvironment and synergistic antitumor effects with a combination of BRAF/MEK inhibition and anti-PD-1 agents. ${ }^{16}$ As a result, many of the current clinical trials for unresectable BRAF-mutant melanoma are examining various combinations of PD-1 or PDL-1 inhibitors with one or more targeted (BRAF/MEK) therapies. ${ }^{17}$

The initial study to evaluate a combination of a BRAF inhibitor (vemurafenib) with immunotherapy (ipilimumab) in 2013 was halted in phase I due to development of severe 
liver toxicity (NCT01400051). ${ }^{18}$ Owing in part to the toxicity observed with ipilimumab in combination with BRAF inhibitors, newer combination studies have focused on PD-1/PDL1 inhibitors. Phase-Ib results were reported from a combination trial of atezolizumab (anti-PDL-1) and vemurafenib in 2015 , demonstrating an overall response rate of $76 \%$ (58\% partial response and $17 \%$ complete response) with median duration of response 20.9 months and no severe adverse events observed (NCT01646442). ${ }^{19}$

Multiple recent clinical trials are examining a triple combination regimen with anti PD-1/PDL-1 agent, BRAF inhibitor, and a MEK inhibitor. The anti-PDL-1 durvalumab was evaluated in combination with dabrafenib and trametenib in a phase-I trial of patients with advanced BRAF-mutant melanoma that completed in 2018 (NCT02027961). ${ }^{20}$ The overall response rate was $69 \%$, and dose-limiting toxicity (reversible grade 3 thrombocytopenia) was observed in one out of 26 patients on the triple therapy regimen. Phase-I data were reported in 2017 from the KEYNOTE-022 study of pembrolizumab, dabrafenib, and trametenib in 15 patients with BRAF-mutant stage III/IV melanoma. ${ }^{21}$ The overall response rate was $67 \%$, and doselimiting toxicities (grades 3 and 4 increase in aminotransferases and grade- 4 neutropenia) were reported in 3 of 15 patients and resolved after drug discontinuation. Phase II of KEYNOTE-022 compared dabrafenib, trametenib, and pembrolizumab to dabrafenib and trametenib in a total of 120 patients with BRAF-positive melanoma (60 per arm; NCT02130466). ${ }^{22}$ The triple therapy arm had significantly higher rates of grade 3 to 5 treatment related adverse events ( 58 vs. $27 \%$ ) and premature discontinuation due to TRAEs was $40 \%$ in the triple therapy arm compared with $20 \%$ for the dabrafenib/trametenib arm. The mean PFS was 16.0 months in the triple therapy group compared with 10.3 months in the dabrafenib/trametenib cohort, which was nonsignificant due to failure to meet one of the parameters for significance (hazard ratio $<0.66$ ) and the median response duration was 18.7 months in the triple therapy group compared with 12.5 months for the dabrafenib/trametenib group. ${ }^{22}$

The IMPemBra trial is a phase-II study comparing pembrolizumab monotherapy to pembrolizumab plus intermittent short-term targeted treatment with dabrafenib and trametenib for 1,2 , or 6 weeks to evaluate safety and tolerability (NCT02625337). ${ }^{23}$ Preliminary results have demonstrated increased overall response rates in the short-term BRAF/ MEK cohorts compared with pembrolizumab monotherapy (objective response rate [ORR] at 18 weeks was $57 \%$ in the pembrolizumab monotherapy group, $71 \%$ in the cohort receiving dabrafenib and trametenib $\times 1$ week, and $83 \%$ in the cohort receiving dabrafenib/trametenib $\times 2$ weeks).

The ongoing COMBI-I trial is a phase-III study evaluating dabrafenib and trametenib with and without the PD-1 inhibitor spartalizumab (NCT02967692). ${ }^{24}$ The COMBI-I study is comprised of three distinct groups: a safety run-in, biomarker analysis with collection of tumor samples for immunohistochemistry, and a randomized double blind placebo-controlled phase to assess efficacy and safety of the dabrafenib and trametenib with and without spartalizumab. Preliminary results reported in 2018 described grade $3 / 4$ adverse events occurring in
$22 \%$ of patients but none led to treatment discontinuation. Significant increases in intratumoral CD8+ lymphocyte infiltration were observed after 2 to 3 weeks of therapy in eight out of nine patients, and the results of the PDL-1 expression and peripheral blood analysis will be reported at a later date. All seven patients evaluated at week 12 demonstrated an unconfirmed partial response, with no patients experiencing disease progression $^{24}$

A phase-II trial of dabrafenib, trametenib, and the PD-1 inhibitor nivolumab is ongoing in patients with unresectable stage III and mestastatic stage IV BRAF-mutant melanomas (NCT02910700). ${ }^{25}$ Preliminary results have been reported in 14 patients thus far, with 3 patients $(21 \%)$ requiring drug discontinuation due to severe adverse events (one case of grade 3 immune-mediated hepatitis and two cases of immunemediated nephritis). Eleven patients have been assessed for response thus far, with 10 patients (91\%) showing a partial response and 1 patient experiencing disease progression. ${ }^{25}$

A triple combination of the PDL- 1 inhibitor atezolizumab, vemurafenib, and cobimetanib has been assessed in a recent phase Ib open-label study (NCT01656442). ${ }^{26,27}$ Enrolled patients had a 28-day lead-in phase with cobimetanib + vemurafenib followed by addition of atezolizumab. The confirmed ORR in 39 patients was $71.8 \%$. The median response duration was 17.4 months, with ongoing response in $39.3 \%$ of patients after 29.9 months of follow-up. ${ }^{26}$ Toxicity of the triple combination was reported with $66 \%$ of patients on triple therapy having grade 3/4 TRAEs including elevations in liver transaminases, hypophosphatemia, and hyponatremia. ${ }^{26}$ In addition, a phase-III study (NEO-VC) of atezolizumab, vemurafenib, and cobimetanib is currently enrolling (NCT02303951).

In summary, combination therapy trials with BRAF/MEK and immunotherapy suggest potential synergistic effects with increased intratumoral CD8 + lymphocytes but long-term data on OS, PFS, and durability of responses achieved are not yet available. In addition, combinations of immunotherapy and targeted therapy may increase toxicity of treatment and optimal combinations of therapeutic agents, as well as dosing schedules are unknown at the present time, but hopefully optimal treatment strategies may become more evident as results begin to emerge from the ongoing phase-II and phase-III combination trials.

\section{Sequencing of Targeted Therapy and Immunotherapy} As discussed above, combination studies of targeted therapies with anti-CTLA-4 or anti PD-1/PDL-1 agents may provide increased efficacy but increased toxicity of these combination regimens are a limiting factor. As such, utilizing the targeted therapies and immunotherapies in sequence as opposed to in combination is an area of active investigation, with the goal of potentially harnessing some of the benefits of combination therapy while minimizing toxicity.

There are currently two active clinical trials investigating the optimal sequencing of targeted therapy and immunotherapy agents. ${ }^{17}$ DREAM-SEQ (NCT02224781) is a phase-III trial in which patients are randomized to receive either ipilimumab + nivolumab or dabrafenib + trametenib, then cross over to the other treatment arm at disease progression. 
The SECOMBIT (NCT02631447) study is a phase-II trial composed of the following three cohorts: encorafenib/binimetanib followed by ipilimumab/nivolumab (arm A), ipilimumab/nivolumab followed by encorafenib/binimetnib ( $\operatorname{arm} B$ ), and a "sandwich" arm treated first with encorafenib/ binimetanib $\times 8$ weeks, ipilimumab/nivolumab until disease progression followed by repeat encorafenib/binimetanib.

While data on optimal therapeutic sequencing from randomized controlled trials are pending, several previous retrospective studies have compared the outcomes of sequenced therapy with BRAF/MEK inhibition before or after immunotherapy. A multicenter retrospective study evaluated 274 patients with BRAF-mutant metastatic melanoma and compared outcomes between those who received first-line immunotherapy (high-dose interleukin-2, ipilimumab, nivolumab, or adoptive T-cell therapy) followed by targeted treatment (with trametinib monotherapy, vemurafenib monotherapy, or combined dabrafenib/trametenib) versus the reverse order. ${ }^{28}$ No significant difference in response to targeted therapy was observed whether it was first or second-line; however, for ipilimumab a decreased response was observed in patients receiving ipilimumab after targeted therapy. ${ }^{28}$ Likewise, in a retrospective analysis of a 93-patient cohort from the ipilimumab expanded access program, superior outcomes were observed in patients who were treated with front-line ipilimumab followed by single-agent vemurafenib or dabrafenib (median OS, 14.5 months) compared with patients initially treated with BRAF inhibition followed by ipilimumab (median OS, 9.9 months). ${ }^{29}$ A more recent retrospective study in 114 patients compared the ORR, OS, and PFS in patients treated with either anti-PD-1 agents or BRAF inhibitors first. ${ }^{30}$ It was noted that patients who progressed on PD-1 inhibitors had worse outcomes after transitioning to subsequent BRAF inhibitors compared with patients who had not received anti-PD-1 agents (median OS, 10.6 vs. 40.3 months; median PFS, 5.0 vs. 7.4 months). A similar phenomenon was observed in patients who started anti-PD-1 after progressing on BRAF inhibitor therapy, with poorer outcomes compared with patients who had not been treated with prior BRAF inhibitors (median OS, 8.2 vs 27.6 months; median PFS, 2.8 vs. 10.6 months). The conclusion was that either front-line BRAF inhibitors or anti-PD-1 agents may be effective, regardless of treatment sequence, but there may be a "shared responder" phenotype between BRAF inhibitors and PD-1 agents with a poor response to one modality predicting a less than optimal response to the other. ${ }^{30}$

\section{Intratumoral Immunotherapy}

Involving direct injection of agents to promote tumor cell lysis, the goal of intratumoral immunotherapy is to promote both local and systemic antitumor immune responses while minimizing systemic toxicities. ${ }^{31}$ Talimogene laherparepvec (T-VEC) is a genetically modified herpes-simplex type 1 virus that was the first oncolytic virus approved for unresectable (stage IIIB, IIIC, or IV) melanoma in 2015. T-VEC was engineered and a gene allowing replication in healthy cells (infected cell protein 34.5, ICP34.5) was removed, and granulocyte colony-stimulating factor (G-CSF) was added to stimulate the local immune response (recruitment of dendritic cells and presentation of tumor antigens to cytotoxic Tcells) upon tumor cell lysis to promote a systemic antitumor immune response.

Currently there are multiple trials examining T-VEC in combination with systemic anti-PD-1 agents, as well as investigating additional novel oncolytic viral candidates (CAVATEK and HF clone 10 [HF-10]), both as monotherapy, as well as in combination with immune checkpoint inhibitors for advanced and metastatic melanoma. In addition, there are multiple nonviral oncolytic agents in clinical trials including PV-10 (rose Bengal), SD-101 (toll-like receptor [TLR]-9 agonist), tilsotomod (TLR-9 agonist), and CMP-001 (TLR 7/8 agonist) which are reviewed briefly below.

\section{Oncolytic Viral Therapies}

T-VEC in Combination with Systemic Immunotherapy T-VEC is currently being studied with both systemic ipilimumab, as well as pembrolizumab for unresectable and metastatic melanoma. A recent phase-II trial compared T-VEC plus iplilimumab to ipilimumab monotherapy in unresectable stage IIIBIV melanoma (NCT01740297). ${ }^{32}$ The ORR was $39 \%$ in the combination arm compared to18\% for ipilimumab monotherapy, and responses of visceral lesions that had not been injected were seen in $52 \%$ of the combination arm compared with $23 \%$ of the ipilumumab monotherapy group. Grade 3 and higher adverse events were observed more frequently in the combination arm than for ipilimumab alone ( $45 \mathrm{vs.} 35 \%$ ) but overall the combination was well tolerated. T-VEC has also been evaluated in combination with pembrolizumab in a phase-Ib trial in which patients received local intratumor T-VEC injection $\left(4 \times 10^{6}\right.$ plaque forming units $/ \mathrm{mL}$ ) at week 1 and began pembrolizumab at week 3 coinciding with the second T-VEC injection. ${ }^{33}$ Biopsies were taken of injected tumors at baseline and at week 3 prior to the second T-VEC injection and before pembrolizumab commenced. The overall response rate was $62 \%$ (with $33 \%$ of patients having a complete response), and immunohistochemical analysis of tumors demonstrated increased CD8+ lymphocyte infiltration, as well as elevated PDL-1, and interferon (IFN)- $\gamma$ expression after the initial T-VEC injection in patients who responded to combination therapy, suggesting that T-VEC may alter the tumor microenvironment to improve the efficacy of PD-1 inhibition. ${ }^{33} \mathrm{~A}$ phase-III trial of T-VEC and pembrolizumab is currently underway (NCT02263508).

\section{Coxsackievirus A21}

Coxsackievirus A21 (CAVATEK; Merck \& Co.) is an unaltered coxsackie virus that preferentially infects cells that express increased levels of intercellular adhesion molecules (ICAMs) on their cell surface. ${ }^{34}$ In the phase-II open-label CAVATEK in melanoma (CALM) study (NCT01227551), 57 patients with stage IIIC-IV melanoma were treated with intratumoral injection of CAVATEK on days $1,3,5$, and 8 followed by a fifth injection 2 weeks later (day 22) and additional injections every 3 weeks (up to a maximum of 10 injections or confirmed disease progression). At 6 months, the ORR was $28 \%$ and no grade 3 or 4 adverse events were reported. ${ }^{35}$ 
CAVATEK was studied in combination with ipilimumab in the phase-Ib study titled melanoma intertumoral CAVATEK and ipilimumab (MITCI; NCT02307149). ${ }^{36}$ The 2017 interim results report in 18 evaluable patients showed an overall response rate of $50 \%$, and notably the response rate was higher in patients who had not been previously treated with a checkpoint inhibitor in the past (60\%) compared with $38 \%$ in patients who had been previously treated with checkpoint inhibitors. However, responses were seen in patients who had developed progressive disease on previous checkpoint inhibitor therapy. In addition, CAVATEK is currently in phaseI trials in combination with pembrolizumab (NCT02307149) and results have not yet been reported.

\section{HF-10 (spontaneously mutated HSV-1)}

HF-10 (Takara Bio Inc.) is a herpes simplex type 1 (HSV-1) virus containing spontaneous mutations (not genetically engineered) that is spontaneously mutated and acts as a potent oncolytic agent. ${ }^{37} \mathrm{~A}$ phase-II trial in combination with ipilimumab has been completed in 44 patients with stage IIIB-IV melanoma (NCT02272855). ${ }^{38}$ The overall response rate at 24 weeks was $41 \%$, with complete responses occurring in $16 \%$ of patients. At 24 weeks, $42.9 \%$ of responses had been maintained without progression. HF-10 is also being evaluated in a phase-II trial with nivolumab for unresectable stage IIIB-IV melanoma (NCT03259425).

\section{Nonviral Oncolytics}

\section{PV-10 (Rose Bengal Disodium)}

PV-10 (Provectus Biopahrmaceuticals Inc.) is a an injectable form of rose Bengal disodium (a xanthene dye) that accumulates in lysosomes after intratumoral injection leading to tumor lysis, and it has received orphan drug designation from the U. S. Food and Drug Administration (FDA) for investigation in melanoma, as well as hepatocellular carcinoma. ${ }^{39} \mathrm{~A}$ phase-II study of PV-10 was recently completed in 80 patients with refractory metastatic melanoma (NCT005211053). The overall response rate was $51 \%$, with a complete response being observed in $26 \%$ of patients. The main side effects were injection site tenderness and itching, and no grade 4 or 5 adverse events were observed. ${ }^{40}$ Intralesional PV-10 is also being evaluated in a phase Ib/II study in combination with pembrolizumab (NCT02557321), as well as a phase-III study, comparing intralesional PV-10, intralesional T-VEC, and systemic chemotherapy with dacarbazine or temozolamide in BRAF-wildtype melanoma who have failed at least one checkpoint inhibitor or are not candidates for checkpoint inhibitor therapy (NCT02288897).

\section{Toll-Like Receptor Agonists}

An important component of immunity, TLRs are transmembrane receptors expressed on a variety of leukocytes including dendritic cells, macrophages, NK cells, T-cells, and B-cells in addition to epithelial and endothelial cells ${ }^{41}$ that are involved in recognition of highly conserved antigens (such as those derived from bacteria, fungi, and viruses) and initiating the innate and adaptive immune responses. TLR agonists have shown potential utility in cancer treatment, by triggering T-cell responses that can lead to antitumor effects. ${ }^{42,43}$ Several TLR agonists have been studied in melanoma, including SD101 (a TLR-9 agonist), tilsotolimod (also known as IMO-2125, a TLR-9 agonist), and NKTR-262 (a TLR 7/8 agonist). ${ }^{31}$ SD-101 (Dynavax Technologies) has been evaluated in a phase-Ib trial in conjunction with pembrolizumab for unresectable and metastatic melanoma (NCT02521870). Patients naïve to previous PD-1/PDL-1 therapy had a significantly better overall response rate than those who had previously had treatment with another PD-1/PDL-1 agent (ORR, 88 vs. 15\%). In addition, immunohistochemical analysis of tumors was performed and an increase in CD8 + T cells, NK cells, dendritic cells, and B-cells was observed in both the PD-1 naïve and previously treated cohorts. ${ }^{44}$ Tilsotolimod IMO-2125 (Idera Pharmaceuticals) has been evaluated in combination with ipilimumab in a phase I/II study in patients with PD-1 refractory metastatic melanoma, and demonstrated an overall response rate of $38 \%$ (NCT02644967). ${ }^{45}$ In addition, tilsotolimod is also being evaluated in combination with ipilimumab for metastatic PD-1 refractory melanoma in the phase-III ILLUMINATE-301 study (NCT03445533). Finally, NKTR-262 is a TLR 7/8 agonist that is currently being evaluated in the phase I/II REVEAL trial in advanced melanoma, MCC, colorectal cancer, urothelial carcinoma, and sarcoma in combination with NKTR-214 (a systemic CD-122 agonist) with or without nivolumab (NCT03435640).

\section{Merkel Cell Carcinoma}

Similarly to melanoma, the systemic treatment of MCC has changed dramatically with the development of anti-PD-1/ PDL-1 immunotherapies. ${ }^{46}$ Avelumab and pembrolizumab are currently approved for metastatic MCC, and current trials are evaluating pembrolizumab, nivolumab, and ipilimumab (in combination with nivolumab) for the treatment of unresectable and metastatic MCC. There are also trials of multiple "next-generation" checkpoint inhibitors targeting the PD-1/ PDL-1 axis including antibodies to T-cell immunoglobulin and mucin containing domain 3 (TIM-3), lymphocyte-activation gene-3 (LAG-3), and T cell immunoreceptor with Ig and immunoreceptor tyrosine-based inhibition motif (ITIM) domains (TIGIT). T-VEC (alone and in combination with nivolumab or radiation therapy) is also being evaluated in MCC, as are several TLR agonists either alone or combined with other immunotherapies. Finally, other immune-mediated treatment mechanisms including adoptive T-cell transfer, cell therapy with innate immune cells, and costimulatory T-cell agonists are in the early phases of trials for MCC.

\section{Pembrolizumab}

The phase II KEYNOTE-017 trial (NCT02267603) evaluated the use of pembrolizumab monotherapy in patients with stage IIIB/IV MCC who had not previously had systemic therapy for their disease. ${ }^{47}$ Results reported in 2018 from 49 patients demonstrated an overall response rate of $56 \%$ (32\% partial and $24 \%$ complete responses) with a median follow-up of 14.9 months. Based on these results, the FDA granted pembrolizumab approval for recurrent or metastatic MCC in 2018, and phase-III trials are currently ongoing to 
assess pembrolizumab as first-line therapy in advanced MCC (NCT03783078).

\section{Nivolumab}

The PD-1 inhibitor nivolumab is currently in phase I/II trials as monotherapy for advanced MCC (CheckMate 358, NCT02488759), and demonstrated an overall response rate of $64 \%$, as well as PFS of $75 \%$ at 6 months. ${ }^{48}$ In addition, the ongoing CheckMate 358 study is also investigating nivolumab in combination for metastatic MCC (phase II), as well as neoadjuvant therapy in resectable disease and in combination with daratumumab (anti-CD38 antibody, phase I) for unresectable or metastatic disease. There are several additional trials of nivolumab in the recruiting phase for MCC, including phase II in combination with ipilimumab and stereotactic radiation for metastatic MCC (NCT03071406), a phase-II trial of nivolumab or ipilimumab compared with placebo following resection of MCC (ADMEC-O, NCT03271372), as well as nivolumab + radiation therapy versus nivolumab, and ipilimumab following resection of MCC (NCT03798639).

\section{“Next-Generation” Checkpoint Inhibitors}

In addition to the inhibitory effect that PD-1/PDL-1 interaction has on the immune response, the tumor microenvironment contains several other inhibitory factors expressed by T-cells including LAG-3 and TIGIT. ${ }^{46}$

\section{LAG-3}

LAG-3 expression may be increased in activated CD8+ and CD4+ T-cells in the setting of chronic infections or malignancy, functioning analogous to PD-1 and suppressing the immune response. ${ }^{49}$ A monoclonal antibody to LAG-3 (INCAGNN02385) is currently being investigated in a phase-I study of advanced tumors including MCC (NCT03538028), and in one of the arms of the CheckMate 358 study evaluating a combination of nivolumab and relatamib (anti-LAG-3 antibody).

\section{TIM-3}

TIM-3 is expressed by CD8+ T-cells within the tumor microenvironment and has been shown to promote T-cell exhaustion, representing a potential target for immunotherapeutic agents based on restoration of cytotoxicity following TIM-3 blockade in vitro. ${ }^{50} \mathrm{~A}$ monoclonal antibody against TIM-3 (INCAGN02390) is currently in phase-I trials for advanced tumors including MCC (NCT03652077).

\section{TIGIT}

TIGIT is a coinhibitory receptor expressed on T-cells and NK cells, and binding to its ligand (CD155) results in dampening of immune response in a manner analogous to PD- $1 .^{51}$ An antiTIGIT antibody (AB154) is currently in phase-I trials both alone and in combination with a novel andi-PD1 antibody (AB154) in advanced tumors including MCC (NCT03628677).

\section{T-VEC}

T-VEC is currently in phase-I trials for locally advanced MCC (NCT03458117), as well as phase-II studies in combination with radiation therapy for unresectable stages III/IV MCC (NCT02819843), as well as nivolumab (NCT02978625).

\section{Toll-Like Receptor Agonists}

Intratumoral injection of TLRs is currently being investigated as adjuvant agents along with checkpoint inhibitors in MCC. AST008 is a TLR-9 agonist in phase-I/II trials in combination with pembrolizumab (NCT0364785). Another phase-I/II study is evaluating NKTR262 (A TLR 7/8 agonist) in combination with nivolumab and systemic NKTR214 (a CD122 agonist) in locally advanced and metastatic MCC (NCT03435640). Finally, polyICLC (polyiosinic-polycytidylic acid with polylysine and carboxymethylcellulose, a TLR-3 agonist) is being investigated in phase-I/II trials in combination with durvalumab (anti-PDL-1 antibody) and tremilumimab (anti-CTLA-4 antibody; (NCT02643303).

\section{Adoptive T-Cell Immunotherapy}

Adoptive T-cell immunotherapy involves the extraction of Tcells followed by subsequent selection and expansion of T-cells against a specific antigen, followed by reinfusion into the patient, and is being investigated as an adjuvant agent in the treatment of MCC using T-cells specific to the Merkel's cell polyomavirus (MCPyV). ${ }^{52} \mathrm{~A}$ phase-I/II trial is evaluating adoptive T-cell therapy plus interferon versus avelumab and radiation therapy in MCPyV-positive unresectable MCC (NCT02584829), and another phase-I/II trial of MCPyV-specific autologous T-cells in combination with avelumab and radiation therapy is slated to begin recruiting (NCT03747484).

\section{T-Cell Costimulation}

T-cell activation involves binding of the TCR to a major histocompatibility complex (MHC) on the antigen presenting cell (APC) in addition to a second signal provided by interaction of costimulatory molecules on the T-cell and APC, and agonists for these costimulatory molecules are an additional adjuvant therapy in development for multiple cancers. ${ }^{53} \mathrm{CD} 27, \mathrm{OX} 40,4$ to $1 \mathrm{BB}$, and glucocorticoid-induced tumor necrosis factor (TNF) receptor-related protein (GITR) are all members of the TNF receptor superfamily and represent costimulatory ligands currently under investigation. ${ }^{54} \mathrm{~A}$ phase-I trial is investigating INCAGN01949 (an anti-OX40 agonistic antibody) in combination with nivolumab, ipilimumab, or both in the treatment of unresectable stage III/IV MCC (NCT03241173). INCAGN01876 is an anti-GITR agonistic antibody currently in two phase-I/II trials for MCC; in combination with nivolumab, ipilimumab, or both (NCT03126110) and in combination with pembrolizumab and epacadostat (inhibitor of indoleamine 2,3 dioxygenase 1 [IDO-1], which acts to suppress effector T-cell function; NCT03277352). In addition, ABBV-368 (an OX40 agonist) is under investigation in combination with ABBV-181 (an antiPD-1 antibody) in a phase-I trial (NCT03071757).

\section{Innate Immune Cell Therapy}

Increased NK cells within the Merkel's cell tumor microenvironment has been shown to be associated with better 
prognosis in MCPyV-associated tumors, ${ }^{55}$ and infusions of activated NK-92 (aNK) cells are being studied both alone and in combination with other agents in MCC. Infusions of Neukoplast (QUILT-3.009 NK cell line) are currently being evaluated in a phase-I single-arm open trial for unresectable stage III-IV MCC (NCT02465957).

\section{Cutaneous Squamous Cell Carcinoma}

Prior to the development of immune checkpoint inhibitors, treatment options for unresectable or metastatic cSCC and systemic treatments were limited to traditional chemotherapy (mainly with platinum-based agents, 5-flurouracil derivatives, doxorubicin, bleomycin, and taxanes) ${ }^{56}$ and agents targeting the epidermal growth factor receptor (EGFR) such as cetuximab, erlotinib, and gefitinib. ${ }^{57}$ In 2018 , cemiplimab became the first anti-PD-1 agent approved for metastatic and unresectable cSCC. $^{58}$ Currently, there are clinical trials investigating cemiplimab as neoadjuvant therapy (either intratumoral or systemic) for recurrent cSCC prior to surgery, as well as an adjuvant agent after surgery, and radiation therapy in high-risk cSCC. In addition, there are multiple clinical trials of other anti-PD-1 agents for first-line treatment of unresectable or metastatic cSCC including pembrolizumab, nivolumab, avelumab, and cosibelimab (CK-301) which is an investigational anti-PDL-1 monoclonal antibody.

\section{Cemiplimab}

The approval of cemiplimab in 2018 for metastatic or unresectable cSCC was based on results from a phase-II study, demonstrating a $47 \%$ overall response rate and a response duration exceeding 6 months in 57\% of patients who responded to cemiplimab (NCT02760498). In addition, cemiplimab was overall well-tolerated with the most common adverse events being diarrhea, fatigue, and nausea, and 7\% of patients needed to discontinue cempilimab due to a TRAE. ${ }^{59}$ Systemic cemiplimab is being evaluated as neoadjuvant therapy prior to surgical resection in stage II-IV cSCC (NCT04154943). Neoadjuvant intralesional cempiplimab is currently being studied in a phase-I trial for recurrent cSCC prior to surgical resection (NCT03889912). In addition, cempiplimab is being evaluated as adjuvant treatment after surgery and radiation for high-risk cSCC compared with placebo (NCT03969004).

\section{Other Anti-PD-1/PDL-1 Agents (Pembrolizumab, Nivolumab, Avelumab, and Cosibelimab/CK-301)}

Pembrolizumab and nivolumab were both approved in 2017 for the first-line treatment of unresectable or metastatic head and neck SCC; however, neither the phase-III study of nivolumab (Checkmate 041) nor the phase-Ib trial of pembrolizumab (KEYNOTE-012) evaluated any patients with cSCC. $^{60,61}$ However, case series of both pembrolizumab and nivolumab have shown promising results in cSCC not amenable to surgical resection and both drugs are currently being evaluated in multiple clinical trials. ${ }^{62,63}$
Pembrolizumab monotherapy is currently being evaluated as first-line treatment for advanced cSCC in four phase-II studies including the KEYNOTE-629 trial (NCT03284424), the CARSKIN trial (NCT02883556) and two additional trials in unresectable or metastatic cSCC (NCT02964559 and NCT02721732). Pembrolizumab is also being studied in combination with an intratumoral TLR-9 agonist (AST-008) in a phase-1b/II trial for advanced cSCC (NCT03684785), as well as an adjuvant agent compared with placebo, following resection of locally advanced cSCC (KEYNOTE-630, NCT03833167). In addition, a phase-I trial of pembrolizumab and the MG1MAGEA3 vaccine (a genetically modified form of the Maraba virus which acts as an oncolytic, coupled with the melanoma antigen family A3 [MAGE-3] antigen that is overexpressed in many tumor types including $\mathrm{CSCC}$ ) is slated to begin recruiting (NCT03773744). ${ }^{64}$

First-line nivolumab is currently being studied in two phase-II trials in locally advanced and metastatic cSCC (NCT03834233 and NCT04204837). Nivolumab is also being evaluated for unresectable and metastatic cSCC in kidney transplant patients in a phase-I trial (NCT03816332) given in conjunction with tacrolimus and prednisone, and patients who have progressive disease on nivolumab will be treated with combination nivolumab and ipilimumab.

There are currently two phase-II studies evaluating the anti-PDL-1 antibody avelumab in unresectable and metastatic cSCC: with and without the anti-EGFR antibody cetuximab (NCT03944941) and in combination with radical radiotherapy (NCT03737721).

Cosibelimab (CK-301) is an investigational anti PDL-1 monoclonal antibody, and is currently being evaluated in a phase-I trial in multiple types of advanced and metastatic cancers including CSCC, MCC, urothelial carcinoma, and both smallcell and non-small-cell lung adenocarcinoma (NCT03212404).

\section{Basal Cell Carcinoma}

While the vast majority of BCC tumors can be treated primarily with surgery and have an excellent prognosis, approximately $1 \%$ of patients develop advanced disease, defined as either locally advanced BCC (laBCC) or metastatic $\mathrm{BCC}(\mathrm{mBCC})$. Though the incidence of $\mathrm{mBCC}$ is extremely rare $(0.0028-0.5 \%)$, it is associated with a median survival of approximately 8 months when nodal metastases are present. ${ }^{65}$ Approximately $85 \%$ of BCCs have mutations in the hedgehog signaling cascade, a pathway critical for cell proliferation and differentiation in embryogenesis, and the treatment of advanced and metastatic BCC has been revolutionized by the introduction of targeted agents inhibiting this pathway. ${ }^{65,66}$ Vismodegib is an inhibitor of the SMO receptor involved in the hedgehog pathway and was approved for advanced BCC in 2012. The approval of vismodegib was based on ERIVANCE, a multicenter, single-arm, twocohort phase-2 trial that assessed the efficacy and safety of vismodegib in patients with laBCC. In this study, 104 patients with laBCC and mBCC were treated with vismodegib $150 \mathrm{mg}$ daily. At 39 months, investigator-assessed ORR was $60.3 \%$ in the laBCC group and $48.5 \%$ in the $\mathrm{mBCC}$ group. Median 
duration of response was 26.2 months in laBCC and 14.8 months in $\mathrm{mBCC}$, demonstrating the durability of response of this treatment option. ${ }^{67}$ Sonidegib is another orally dosed SMO inhibitor that is structurally distinct from vismodegib, and was approved in 2015 for locally advanced BCC. In the double-blind phase-2 BOLT trial, patients with laBCC or $\mathrm{mBCC}$ were randomized to either 200 or $800 \mathrm{mg}$ of sonidegib daily. No additional efficacy was found between the two doses and in the 200-mg group, the ORR performed by central review was $56.1 \%$ for laBCC and $7.7 \%$ for $\mathrm{mBCC}$ at 30-month follow-up, with the 200-mg group also exhibiting a better safety profile. ${ }^{68}$

To date, there are no head-to-head randomized controlled trials comparing vismodegib to sonidegib. In a meta-analysis of 1,102 patients, vismodegib and sonidegib demonstrated a similar overall response rate (RR) for laBCC (69 vs. 57\%, respectively), whereas the complete RR differed drastically (31 vs. 3\%, respectively). For metastatic disease, the overall RR of vismodegib was 2.7-fold higher than that of sonidegib (39 vs. $15 \%$, respectively). ${ }^{69}$ However, there is a need for future head-to-head clinical trials to further compare the efficacy and safety profiles of these two agents.

Current areas of research in BCC include the use of SMO inhibitors as neoadjuvant agents prior to surgical resection of locally advanced BCC, topical SMO inhibitors (patidegib) for prevention and treatment of BCCs in the setting of BCC nevus syndrome (BCCNS) resulting from Patched-1 mutations, as well as to treat and prevent high-frequency BCCs in patients who do not have BCCNS, investigation of novel SMO inhibitors, as well as alternative targets for inhibition within the hedgehog pathway.

\section{Novel Smoothened Inhibitors}

Taladegib is an investigational oral SMO inhibitor that recently completed a phase dose-finding I clinical trial for advanced BCC (NCT01226485). Data from the phase-I study reported an overall response rate of $46.8 \%$ (in both patients who had previous hedgehog pathway inhibitors as well as hedgehognaïve patients) with an acceptable safety profile. ${ }^{70}$ Phase-II trials are planned at doses below the maximum tolerated dose (MTD) of $400 \mathrm{mg}$ daily that was identified in the phase-I study.

\section{Neoadjuvant Smoothened Inhibitors}

Neoadjuvant SMO inhibitors for laBCC have been investigated, specifically for BCCs at high risk for functional or aesthetic compromise with surgery in an effort to decrease the size of the defect required for tumor clearance. Current evidence for their use in the neoadjuvant setting is not well established, based primarily on open-label studies and case series. In an open-label study in 15 patients, vismodegib $150 \mathrm{mg}$ daily was used for 3 to 6 months in the neoadjuvant setting, with a reduction of surgical defect size by $27 \%$ (95\% confidence interval $[\mathrm{CI}]:-45.7$ to $-7.9 \%, p=0.006)$. Four patients were not able to continue vismodegib longer than 3 months due to side effects, and 1 patient out of 11 evaluable patients had recurrence after a mean follow-up of 11.5 months. ${ }^{71}$ However, it is important to note that systemic SMO inhibitors do not always reduce tumor volume in a concentric fashion from the periphery, and may result in a tumor that effectively has "skip" lesions and reduce the accuracy of microscopically controlled excision methods such as Mohs' surgery. Larger randomized placebo-controlled studies will be required to investigate the efficacy of neoadjuvant SMO inhibitors for advanced BCC.

\section{Topical Smoothened Inhibitors}

With more than one quarter of patients discontinuing treatment due to side effects, the use of topical SMO inhibitors is currently being explored. In a double-blind, vehicle-controlled, intraindividual study, 8 nevoid BCC patients with 27 BCCs were randomized to twice daily treatment of either $0.75 \%$ sonidegib cream $(n=13)$ or vehicle $(n=14)$. In the topical sonidegib group, three lesions demonstrated a complete response, and nine had a partial response (PR); only one PR was noted in the placebo group. ${ }^{72}$ Topical patidegib is currently being studied for its ability to decrease BCC disease burden in the setting of BCCNS (phase III, NCT03703310) as well as in non-BCCNS high-frequency BCCS (phase II, NCT04155190).

\section{Alternative Targets in the Hedgehog Pathway}

In addition to side effects, resistance to SMO inhibitors raises a major concern, as approximately 5 to $10 \%$ of patients demonstrate resistance with eventual lack of response and progression of disease. ${ }^{65}$ The use of additional therapies directed at alternative downstream targets of the hedgehog signaling pathway have been considered for cases of resistance. Iraconazole, an FDA-approved antifungal, inhibits the hedgehog pathway by blocking SMO migration and has been shown to inhibit GLI expression in cells with vismodegib-resistant mutations. A clinical trial investigating the use of oral SUBA-itraconazole (SUBA-Cap) for BCCNS is currently in phase II (NCT02354261). Topical itraconazole is also in a phase-I study for non-BCCNS patients with BCC (NCT02735356). Additionally, direct inhibition of GLI transcription factors, terminal effectors in the hedgehog pathway, may also be a promising target in the treatment of laBCCs. Arsenic trioxide (ATO) is a chemotherapeutic agent that directly binds GLI1 and GLI2, preventing the accumulation of $G L I$ in the primary cilium, an essential step in downstream hedgehog signaling. ${ }^{73}$ While combination therapies targeting more than one component of the hedgehog pathway may be more effective in combating resistance, future clinical studies are necessary for further confirmation.

\section{Immunotherapy (PD-1 Inhibitors)}

Pembrolizumab is currently in phase-II clinical trials for advanced BCC in combination with vismodegib (NCT02690948). Phase-I results were recently reported in which 16 patients were assigned to either pembrolizumab monotherapy versus pembrolizumab with oral vismodegib. Although not randomized, the authors concluded that the overall response rate of the pembrolizumab-vismodegib combination group was not subjectively superior compared with the pembrolizumab monotherapy group but merits further 
investigation in larger randomized controlled trials. ${ }^{74}$ Cepilimumab monotherapy is currently in phase-II trials for in patients with BCC that had either progressed on or been intolerant prior hedgehog inhibitor therapy (NCT03132636) and results have not yet been published. ${ }^{75}$ However, a case report in one patient with metastatic BCC resistant to hedgehog inhibitors had a partial response with cepilimumab, and PFS was 32 weeks. ${ }^{76}$ Nivolumab has not been studied in clinical trials but has shown a partial response in a case report of one patient with metastatic BCC refractory to hedgehog inhibitors and a PFS time of 116 weeks. $^{77}$

\section{Conclusion}

The development of immunotherapeutic agents targeting the PD-1/PDL-1 axis has dramatically altered the treatment of and improved survival outcomes of many cancers that previously had limited therapeutic options in advanced stages, including melanoma, MCC, and cSCC. Numerous other treatments have emerged for melanoma, including the development of targeted agents against the BRAF/MEK pathway, as well intratumoral oncolytic agents, which are being investigated both alone and in combination with immunotherapy. Trials exploring various combinations and administration sequences of approved targeted therapies and immune-based treatments are underway in melanoma in an effort to help improve efficacy while minimizing toxicity. In addition, the treatment of advanced and metastatic BCC has been significantly improved by the development of targeted inhibitors of the hedgehog signaling pathway, and future directions include investigation of PD- 1 inhibitors as a means to augment response and potentially treat BCCs that are resistant to hedgehog inhibitors. With multiple late-stage clinical trials underway, the next several years will provide valuable data on safety, efficacy, and durability of responses achieved by these recently approved targeted and immunebased therapies, as well as hopefully provide insight into the optimal treatment combinations, and regimens for advanced melanoma and non-melanoma skin cancers.

\section{Conflict of Interest}

J.A.C. reports grants from Regeneron, outside the submitted work. M.L.S. reports and coinvestigator on intralesional cepilipumab for recurrent SCC. All the other authors report no conflict of interest.

\section{References}

1 Aderhold K, Wilson M, Berger AC, Levi S, Bennett J. Precision medicine in the treatment of melanoma. Surg Oncol Clin N Am 2020;29(01):1-13

2 Long GV, Hauschild A, Santinami M, et al. Adjuvant dabrafenib plus trametinib in stage III BRAF-mutated melanoma. $\mathrm{N}$ Engl J Med 2017;377(19):1813-1823

3 Ascierto PA, McArthur GA, Dréno B, et al. Cobimetinib combined with vemurafenib in advanced $B R A F(V 600)$-mutant melanoma (coBRIM): updated efficacy results from a randomised, doubleblind, phase 3 trial. Lancet Oncol 2016;17(09):1248-1260

4 Dummer R, Ascierto PA, Gogas HJ, et al. Overall survival in patients with BRAF-mutant melanoma receiving encorafenib plus binimetinib versus vemurafenib or encorafenib (COLUMBUS): a multicentre, open-label, randomised, phase 3 trial. Lancet Oncol 2018;19(10):1315-1327

5 Cohen JV, Buchbinder EI. The evolution of adjuvant therapy for melanoma. Curr Oncol Rep 2019;21(12):106

6 Eggermont AM, Chiarion-Sileni V, Grob JJ, et al. Prolonged survival in stage III melanoma with ipilimumab adjuvant therapy. N Engl J Med 2016;375(19):1845-1855

7 Snyder A, Makarov V, Merghoub T, et al. Genetic basis for clinical response to CTLA-4 blockade in melanoma. N Engl J Med 2014; 371(23):2189-2199

8 Coens C, Suciu S, Chiarion-Sileni V, et al. Health-related quality of life with adjuvant ipilimumab versus placebo after complete resection of high-risk stage III melanoma (EORTC 18071): secondary outcomes of a multinational, randomised, double-blind, phase 3 trial. Lancet Oncol 2017;18(03):393-403

9 Tarhini AA, et al. Phase III study of adjuvant ipilimumab (3 or $10 \mathrm{mg} / \mathrm{kg}$ ) versus high-dose interferon alfa-2b for resected highrisk melanoma: North American Intergroup E1609. J Clin Oncol 2020;38(06):567-575

10 Keir ME, Butte MJ, Freeman GJ, Sharpe AH. PD-1 and its ligands in tolerance and immunity. Annu Rev Immunol 2008;26:677-704

11 Dong H, Strome SE, Salomao DR, et al. Tumor-associated B7-H1 promotes T-cell apoptosis: a potential mechanism of immune evasion. Nat Med 2002;8(08):793-800

12 Akinleye A, Rasool Z. Immune checkpoint inhibitors of PD-L1 as cancer therapeutics. J Hematol Oncol 2019;12(01):92

13 Keilholz U, Mehnert JM, Bauer S, et al. Avelumab in patients with previously treated metastatic melanoma: phase $1 \mathrm{~b}$ results from the JAVELIN Solid Tumor trial. J Immunother Cancer 2019;7(01):12

14 Robert C, Karaszewska B, Schachter J, et al. Improved overall survival in melanoma with combined dabrafenib and trametinib. N Engl J Med 2015;372(01):30-39

15 Ugurel S, Röhmel J, Ascierto PA, et al. Survival of patients with advanced metastatic melanoma: the impact of novel therapiesupdate 2017. Eur J Cancer 2017;83:247-257

16 Reddy SM, Reuben A, Wargo JA. Influences of BRAF inhibitors on the immune microenvironment and the rationale for combined molecular and immune targeted therapy. Curr Oncol Rep 2016;18 (07):42

17 Pavlick AC, Fecher L, Ascierto PA, Sullivan RJ. Frontline therapy for BRAF-mutated metastatic melanoma: how do you choose, and is there one correct answer? Am Soc Clin Oncol Educ Book 2019; 39:564-571

18 Ribas A, Hodi FS, Callahan M, Konto C, Wolchok J. Hepatotoxicity with combination of vemurafenib and ipilimumab. N Engl J Med 2013;368(14):1365-1366

19 Hamid O, Hodi S. Preliminary clinical safety, tolerability, and activity of atezolizumab (anti-PD-L1) combined with vemurafenib in BRAF V600 metastatic melanoma, in Presented at Society for Melanoma research 2015 Congress October 2015 San Francisco CA. 2015

20 Ribas A, Butler M, Lutzky J, et al. Phase I study combining antiPDL-1 (MEDI4736) with BRAF (dabrefenib) and.or MEK (trametenib) inhibitors in advanced melanoma. J Clin Oncol 2017;33(15, Suppl):3003-3003

21 Ribas A, Hodi FS, Lawrence D, et al. KEYNOTE-22 update: phase I study of first-line pembrolizumab plus dabrafenib and trametinib for BRAF-mutant advanced melanoma. Ann Oncol 2017;28 (Suppl 5):v428-v448

22 Ascierto PA, Ferrucci PF, Fisher R, et al. Dabrafenib, trametinib and pembrolizumab or placebo in BRAF-mutant melanoma. Nat Med 2019;25(06):941-946

23 Rozeman EA, Deken MA, Gadiot J, et al. Phase II study comparing pembrolizumab (PEM) with intermittent/short-term dual MAPK pathway inhibition (dabrafenib + trametenib) plus pembrolizumab in patients harboring the BRAF V600 mutation (IMPemBra triial). Ann Oncol 2018;29:LBA46 
24 Dummer R, Nathan P. The anti-PD-1 antibody spartalizumab (PDR001) in combination with dabrafenib and trametenib in previously untreated patients with advanced BRAF V600-mutant melanoma: first efficacy, safety and biomarker finsings from the part 2 biomarker cohort of COMBI-i. Cancer Res 2018;78:CT182

25 Tawbi HAH, Glitza IC. Safety and preliminary activity data from a single center phase II study of nivolumab with dabrafenib and trametenib in patients with BRAF-mutated metastatic melanoma. J Clin Oncol 2018;36(15, Suppl):9560-9650

26 Sullivan RJ, Hamid O, Gonzalez R, et al. Atezolizumab plus cobimetinib and vemurafenib in BRAF-mutated melanoma patients. Nat Med 2019;25(06):929-935

27 Sullivan RJ, Lewis KD. Atezolizumab (A) + cobimetanib (C) + vemurafenib (V)in BRAF-mutant metastatic melanoma (mel): updated safety and clinical activity. J Clin Oncol 2017;35:abstr3063

28 Ackerman A, Klein O, McDermott DF, et al. Outcomes of patients with metastatic melanoma treated with immunotherapy prior to or after BRAF inhibitors. Cancer 2014;120(11):1695-1701

29 Ascierto PA, Simeone E, Sileni VC, et al. Sequential treatment with ipilimumab and BRAF inhibitors in patients with metastatic melanoma: data from the Italian cohort of the ipilimumab expanded access program. Cancer Invest 2014;32(04):144-149

30 Johnson DB, Pectasides E, Feld E, et al. Sequencing Treatment in BRAFV600 Mutant Melanoma: Anti-PD-1 Before and After BRAF Inhibition. J Immunother 2017;40(01):31-35

31 Hamid O, Ismail R, Puzanov I. Intratumoral ImmunotherapyUpdate 2019. Oncologist 2019:theoncologist.2019-0438

32 Chesney J, Puzanov I, Collichio F, et al. Randomized, open-label phase II study evaluating the efficacy and safety of talimogene laherparepvec in combination with ipilimumab versus ipilimumab alone in patients with advanced, unresectable melanoma. J Clin Oncol 2018;36(17):1658-1667

33 Ribas A, Dummer R, Puzanov I, et al. Oncolytic virotherapy promotes intratumoral $T$ cell infiltration and improves anti-PD1 immunotherapy. Cell 2017;170(06):1109-1119.e10

34 Xiao C, Bator-Kelly CM, Rieder E, et al. The crystal structure of coxsackievirus A21 and its interaction with ICAM-1. Structure 2005;13(07):1019-1033

35 Andtbacka RCB, Kaufman H, et al. Final data from CALM: a phase II study of coxsackie virus A21 (CVA21) oncolytic virus imunotherapy in patients with advanced melanoma. J Clin Oncol 2015; 33:9030a

36 Curti BRJ, Hallmeyer S, et al. The MITCI (Phase Ib) study: a novel immunotherapy combination of intralesional coxsackie virus A21 and systemic ipilimumab in advanced melanoma patients with or without previous immune checkpoint treatment. Presented at the American Association for Cancer Research Annual Meeting April 1-6, 2017 Washington DC, 2017

37 Eissa IR, Naoe Y, Bustos-Villalobos I, et al. Genomic signature of the natural oncolytic herpes simplex virus hf10 and its therapeutic role in preclinical and clinical trials. Front Oncol 2017;7:149

38 Andtbacka RHI, Agarwala SS, et al. Final results of a phase II multicenter trial of HF10, areplication-competent oncolytic virus, and ipilimumab combination treatment in patients with stage IIIB-IV unresectable or metastatic melanoma. J Clin Oncol 2017;35:9510a

39 Read TA, Smith A, Thomas J, et al. Intralesional PV-10 for the treatment of in-transit melanoma metastases-Results of a prospective, non-randomized, single center study. J Surg Oncol 2018; 117(04):579-587

40 Thompson JF, Agarwala SS, Smithers BM, et al. Phase 2 study of intralesional PV-10 in refractory metastatic melanoma. Ann Surg Oncol 2015;22(07):2135-2142

41 Takeda K, Akira S. Toll-like receptors. Curr Protoc Immunol 2015; 109:1-10, 10

42 Baines J, Celis E. Immune-mediated tumor regression induced by CpG-containing oligodeoxynucleotides. Clin Cancer Res 2003;9 (07):2693-2700
43 Carpentier A, Laigle-Donadey F, Zohar S, et al. Phase 1 trial of a $\mathrm{CpG}$ oligodeoxynucleotide for patients with recurrent glioblastoma. Neuro-oncol 2006;8(01):60-66

44 Ribas A, Medina T, Kummar S, et al. SD-101 in combination with pembrolizumab in advanced melanoma: results of a phase $\mathrm{Ib}$, multicenter study. Cancer Discov 2018;8(10):1250-1257

45 Diab A, Benatchez C, et al. Intratumoral (IT) injection of the tlr9 agonist tilsotolimod (IMO-2125) in combination with ipilumimab triggers durable responses in PD-1 inhibitor refractory metastatic melanoma (rMM): Results from a multicenter, phase I/II study. Ann Oncol 2018;29:442-466

46 Samimi M. Immune checkpoint inhibitors and beyond: an overview of immune-based therapies in merkel cell carcinoma. Am J Clin Dermatol 2019;20(03):391-407

47 Nghiem PT, Bhatia S, Lipson EJ, et al. PD-1 Blockade with pembrolizumab in advanced Merkel-cell carcinoma. N Engl J Med 2016;374(26):2542-2552

48 Topalian SL, Hollebecque A, et al. Non-comparitive, open label, multiple cohort, phase $1 / 2$ atudy to evaluate nivolumab in patients with virus-associated tumors (CheckMate 358 ): efficacy and safety in Merkel cell carcinoma. Cancer Res 2017;77(13, Suppl):CT074

49 Anderson AC, Joller N, Kuchroo VK. Lag-3, Tim-3, and TIGIT: Coinhibitory Receptors with Specialized Functions in Immune Regulation. Immunity 2016;44(05):989-1004

50 Afanasiev OK, Yelistratova L, Miller N, et al. Merkel polyomavirusspecific T cells fluctuate with merkel cell carcinoma burden and express therapeutically targetable PD- 1 and Tim- 3 exhaustion markers. Clin Cancer Res 2013;19(19):5351-5360

51 Solomon BL, Garrido-Laguna I. TIGIT: a novel immunotherapy target moving from bench to bedside. Cancer Immunol Immunother 2018;67(11):1659-1667

52 Chapuis AG, Afanasiev OK, Iyer JG, et al. Regression of metastatic Merkel cell carcinoma following transfer of polyomavirus-specific $\mathrm{T}$ cells and therapies capable of re-inducing HLA class-I. Cancer Immunol Res 2014;2(01):27-36

53 Marin-Acevedo JA, Soyano AE, Dholaria B, Knutson KL, Lou Y. Cancer immunotherapy beyond immune checkpoint inhibitors. J Hematol Oncol 2018;11(01):8

54 Buchan SL, Rogel A, Al-Shamkhani A. The immunobiology of CD27 and OX40 and their potential as targets for cancer immunotherapy. Blood 2018;131(01):39-48

55 Laniosz V, Onajin O, Sominidi-Damodaran S, Meves A, Gibson LE, Baum CL. Natural killer cell response is a predictor of good outcome in $\mathrm{MCPyV}^{+}$Merkel cell carcinoma: a case series of 23 patients. J Am Acad Dermatol 2017;77(01):31-32

56 Sadek H, Azli N, Wendling JL, et al. Treatment of advanced squamous cell carcinoma of the skin with cisplatin, 5-fluorouracil, and bleomycin. Cancer 1990;66(08):1692-1696

57 Guminski A, Stein B. Immunotherapy and other systemic therapies for cutaneous SCC. Oral Oncol 2019;99:104459

58 Markham A, Duggan S. Cemiplimab: first global approval. Drugs 2018;78(17):1841-1846

59 Migden MR, Rischin D, Schmults CD, et al. PD-1 Blockade with Cemiplimab in Advanced Cutaneous Squamous-Cell Carcinoma. N Engl J Med 2018;379(04):341-351

60 Ferris RL, Licitra L, Fayette J, et al. Nivolumab in patients with recurrent or metastatic squamous cell carcinoma of the head and neck: efficacy and safety in CheckMate 141 by prior cetuximab use. Clin Cancer Res 2019;25(17):5221-5230

61 Seiwert TY, Burtness B, Mehra R, et al. Safety and clinical activity of pembrolizumab for treatment of recurrent or metastatic squamous cell carcinoma of the head and neck (KEYNOTE-012): an open-label, multicentre, phase 1b trial. Lancet Oncol 2016;17 (07):956-965

62 Winkler JK, Schneiderbauer R, Bender C, et al. Anti-programmed cell death-1 therapy in nonmelanoma skin cancer. $\mathrm{Br} J$ Dermatol 2017;176(02):498-502 
63 Tran DC, Colevas AD, Chang AL. Follow-up on programmed cell death 1 inhibitor for cutaneous squamous cell carcinoma. JAMA Dermatol 2017;153(01):92-94

64 Pol JG, Acuna SA, Yadollahi B, et al. Preclinical evaluation of a MAGEA3 vaccination utilizing the oncolytic Maraba virus currently in first-in-human trials. Oncolmmunology 2018;8(01):e1512329

65 Leavitt E, Lask G, Martin S. Sonic hedgehog pathway inhibition in the treatment of advanced basal cell carcinoma. Curr Treat Options Oncol 2019;20(11):84

66 Gutzmer R, Solomon JA. Hedgehog pathway inhibition for the treatment of basal cell carcinoma. Target Oncol 2019;14(03): 253-267

67 Sekulic A, Migden MR, Basset-Seguin N, et al; ERIVANCE BCC Investigators. Long-term safety and efficacy of vismodegib in patients with advanced basal cell carcinoma: final update of the pivotal ERIVANCE BCC study. BMC Cancer 2017;17(01):332

68 Lear JT, Migden MR, Lewis KD, et al. Long-term efficacy and safety of sonidegib in patients with locally advanced and metastatic basal cell carcinoma: 30 -month analysis of the randomized phase 2 BOLT study. J Eur Acad Dermatol Venereol 2018;32(03):372-381

69 Xie P, Lefrançois P. Efficacy, safety, and comparison of sonic hedgehog inhibitors in basal cell carcinomas: A systematic review and meta-analysis. J Am Acad Dermatol 2018;79(06):1089-1100.e17

70 Bendell J, Andre V, Ho A, et al. Phase I Study of LY2940680, a Smo Antagonist, in Patients with Advanced Cancer Including Treat-
ment-Naïve and Previously Treated Basal Cell Carcinoma. Clin Cancer Res 2018;24(09):2082-2091

71 Ally MS, Aasi S, Wysong A, et al. An investigator-initiated openlabel clinical trial of vismodegib as a neoadjuvant to surgery for high-risk basal cell carcinoma. J Am Acad Dermatol 2014;71(05): 904-911.e1

72 Skvara H, Kalthoff F, Meingassner JG, et al. Topical treatment of Basal cell carcinomas in nevoid Basal cell carcinoma syndrome with a smoothened inhibitor. J Invest Dermatol 2011;131(08):1735-1744

73 Beauchamp EM, Ringer L, Bulut G, et al. Arsenic trioxide inhibits human cancer cell growth and tumor development in mice by blocking Hedgehog/GLI pathway.J Clin Invest 2011;121(01):148-160

74 Chang ALS, Tran DC, Cannon JGD, et al. Pembrolizumab for advanced basal cell carcinoma: An investigator-initiated, proofof-concept study. J Am Acad Dermatol 2019;80(02):564-566

75 Choi FD, et al. Programmed cell death 1 protein and programmed death-ligand 1 inhibitors in the treatment of nonmelanoma skin cancer: A systematic review. J Am Acad Dermatol 2020;82(02): 440-459

76 Falchook GS, Leidner R, Stankevich E, et al. Responses of metastatic basal cell and cutaneous squamous cell carcinomas to anti-PD1 monoclonal antibody REGN2810. J Immunother Cancer 2016;4:70

77 Ikeda S, Goodman AM, Cohen PR, et al. Metastatic basal cell carcinoma with amplification of PD-L1: exceptional response to anti-PD1 therapy. NPJ Genom Med 2016;1:1 